\title{
Partially saturated canthaxanthin purified from Aspergillus carbonarius induces apoptosis in prostate cancer cell line
}

\author{
Nallasamy Kumaresan • Konasur R. Sanjay • \\ Kundumani S. Venkatesh • Ravi-Kumar Kadeppagari • \\ Govindaswamy Vijayalakshmi • \\ Sukumaran Umesh-Kumar
}

Published online: 15 July 2008

(C) Springer-Verlag 2008

\section{Erratum to: Appl Microbiol Biotechnol 10.1007/s00235-008-1538-7}

Unfortunately, the title and the abstract of the article were published with an error. The spelling of the word 'prostate' was incorrect. The correct title and abstract are given here.

A mutant Aspergillus carbonarius selected for temperature tolerance after UV treatment, when grown in shake flasks, produced mycelia bearing yellow pigment. Since the mutant was affected in sterol biosynthetic pathway, the pigment was apparently produced to maintain membrane fluidity and rigidity for growth sustenance in low $\mathrm{pH}$ culture broth. NMR analyses characterizing the pigment as a partially saturated canthaxanthin, containing $\beta$-ionone end rings, suggested its application as a retinoid. When tested for this property in retinoic acid receptor expressing prostate cancer cell line, LNCaP, the fungal partially saturated canthaxanthin induced apoptosis. Low apoptosis percentage in DU145 prostate cancer cells, that does not express functional RAR- $\beta$ receptor, suggested binding specificity of the partially saturated canthaxanthin for RAR- $\beta$.

The online version of the original article can be found at http://dx.doi. org/10.1007/s00253-008-1538-7.

N. Kumaresan · K. R. Sanjay · G. Vijayalakshmi •

S. Umesh-Kumar $(\bowtie)$

Food Microbiology Department,

Central Food Technological Research Institute,

Mysore 570 020, India

e-mail: umeshkumar@cftri.res.in

Present address:

K. S. Venkatesh • R.-K. Kadeppagari

Department of Microbiology, Immunology and Parasitology,

Stanley S. Scott Cancer Center, LSU Health Sciences Center,

New Orleans, LA 70112-1393, USA 